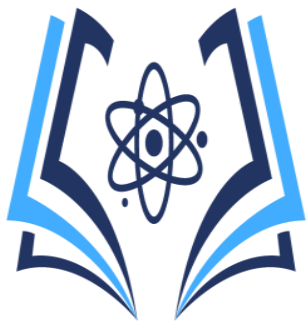

\title{
Hábitos alimenticios en adolescentes de 13 a 19 años en el centro de salud Junín en tiempos de pandemia
} Eating habits in adolescents aged 13 to 19 years at the Junín health center in times of pandemic

\author{
Hábitos alimenticios en adolescentes de 13 a 19 años
}

\author{
Lic. Luigi Antonio Pilay Bravo ${ }^{1}$ \\ Dr. Roberth Zambrano Santos PhD. ${ }^{2}$ \\ ${ }^{1}$ Universidad Estatal del Sur de Manabí, pilay-luigi7776@unesum.edu.ec , ORCID https://orcid.org/0000- \\ 0002-3887-5684 \\ 2 Universidad Estatal del Sur de Manabí, rzambranosantos@yahoo.es, ORCID https://orcid.org/0000-0002- \\ $4072-4738$
}

Correo de contacto: pilay-luigi7776@unesum.edu.ec

Recibido: 20-04-2021

Aprobado: 14-06-2021

\begin{abstract}
Resumen
La investigación se centró en los adolescentes de 13 a 19 años del centro de salud Junín en quienes se evidenció que muestran deficiencias en la práctica de hábitos alimenticos saludables, problemática que se ha acentuado más en tiempos de pandemia por el COVID19. Se estableció como objetivo general identificar los hábitos alimenticios en los adolescentes de 13 a 19 años del centro de salud Junín en tiempos de pandemia, el objeto de estudio fue los hábitos alimenticos. Se determinó como variable independiente: hábitos alimenticios, y variable dependiente: pandemia. El estudio fue descriptivo de cohorte transversal, con la ayuda de los métodos estadísticos se pudo procesar y analizar los datos relacionados con las variables, la población de estudio estuvo conformada por 200 adolescentes de 13 a 19 años de ambos sexos. Los resultados de la investigación mostraron que la mayoría de los adolescentes de 13 a 19 años de edad son de género femenino, con el cuestionario se determinó que la alimentación de los adolescentes durante esta pandemia ha sido. Finalmente se concluye que la mayor parte de alimentos que consumieron los adolescentes durante la pandemia son escaso de vitaminas, minerales y proteínas; es necesario que la dieta sea equilibrada para que aporte los nutrientes necesarios para el desarrollo y crecimiento del adolescente.
\end{abstract}

Palabras claves: Conducta alimentaria, dieta, estilos de vida, pandemia, crisis sanitaria.Abstract
The research focused on adolescents between the ages of 13 and 19 from the Junín health center in whom it was shown that they show deficiencies in the practice of healthy eating habits, a problem that has become more accentuated in times of the COVID19 pandemic. It was established as a general objective to identify the eating habits in adolescents from 13 to 19 years of the Junín health center in times of pandemic, the object of study was eating habits. It was determined as independent variable: eating habits, and dependent variable: pandemic. The study was descriptive of a cross-sectional cohort, with the help of statistical methods it was possible to process and analyze the data related to the variables, the study population consisted of 200 adolescents between 13 and 19 years of age of both sexes. The results of the research showed that the majority of adolescents from 13 to 19 years of age are female, with the questionnaire it was determined that the diet of adolescents during this pandemic has been. Finally, it is concluded that most of the foods that adolescents consumed during the pandemic are lacking in vitamins, minerals and proteins; it is necessary that the diet is balanced so that it provides the necessary nutrients for the development and growth of the adolescent.

Keywords: Eating behavior, diet, lifestyles, pandemic, health crisis.

Introducción

La salud pública tiene un objetivo central, el cual es mantener un buen estado de la salud, el bienestar y el alargamiento de la vida humana con calidad, lo cual demanda una acción integradora (Pacheco, 
Michelena, Mora, \& Miranda, 2014) buscando mejores entornos y estilos de vida para los adolescentes. Una alimentación saludable se establece como uno de los más importantes factores para una adecuada y optima salud que brinde calidad de vida al ser humano, por esta razón una alimentación inadecuada proporciona mayor riesgo de enfermedades o de un desbalance nutricional (Morales, 2020). Los estilos de vida son la base fundamental para la prevención de enfermedades crónicas no transmisibles a futuro.

La Organización Mundial de la Salud acepta como adolescencia a los individuos entre 10 a 19 años de edad; considerando dos fases: la adolescencia temprana de 11 a 14 años y la adolescencia tardía de 15 a 19 años. Esa etapa de vida se considera una fase de transición gradual de la infancia a la edad adulta representando uno de los periodos más importantes del ciclo de la vida del ser humano, pues es el momento en que el crecimiento y el desarrollo se completan y culminan en la plena capacidad reproductiva (Ministerio de Salud Pública del Ecuador, 2012).

La adolescencia es una etapa muy importante en el desarrollo de la persona esto involucra muchos cambios tanto fisiológicos como psicológicos. Estos cambios influyen sobre el comportamiento, necesidades nutricionales y hábitos alimentarios. La adolescencia se caracteriza por un intenso crecimiento y desarrollo fisiológico, hasta el punto que se llega a alcanzar en un periodo relativamente corto de tiempo el $50 \%$ del peso corporal definitivo. En consecuencia, las necesidades de energía y nutrientes van a aumentar en la adolescencia (ALvarado \& Barros, 2016).

El estilo de vida de determinados grupos de población especialmente de los adolescentes puede conducir a hábitos alimentarios y modelos dietéticos, que se comporten como factores de riesgo para la salud y se desencadenen en enfermedades crónicas. Las presiones publicitarias, los regímenes de adelgazamiento programados para adaptarse a la sociedad, los horarios irregulares en cada tiempo de comida, etc. Son factores que pueden convertirse en un riesgo nutricional para los adolescentes (Martínez, Cobo, \& Carbajal, 2011) y más ahora que el mundo entero se ve inmerso en una crisis sanitaria, debido a esto muchas personas han perdido sus trabajos, $y$ por ende su alimentación ha sido la más afectada llevando a sus paladares comidas muy bajas en nutrientes, minerales y proteínas.

Según la Organización Mundial de la Salud, la nutrición es la ingesta de alimentos en relación con las necesidades dietéticas del organismo. Una buena nutrición (una dieta suficiente y equilibrada combinada con el ejercicio físico regular) es un elemento fundamental de la buena salud (ALvarado \& Barros, 2016). Una mala nutrición puede reducir la inmunidad aumentar la
Vol. 4, Nro. 1, Publicado 2021-06-30

vulnerabilidad a las enfermedades, alterar el desarrollo físico y mental y reducir la productividad (Organización Mundial de la Salud, 2016). Por lo tanto, es muy importante realizar la evaluación del estado nutricional de los adolescentes, teniendo en cuenta que la malnutrición tanto por déficit (desnutrición y carencias especificas) o por exceso (obesidad) tiene una alta prevalencia de la adolescencia y que ello condiciona la movilidad y mortalidad en los mismos (PUCE, Escuela de medicina, 2015).

Debido a que la juventud se encuentra en una etapa crucial en el desarrollo de la persona donde se van adquiriendo hábitos que en la mayoría de los casos se mantienen en la edad adulta, estos pueden afectar positiva o negativamente su salud los hábitos de riesgos incrementan de forma notable la probabilidad de desarrollar ciertas patologías en la vida adulta sobre todo generando trastornos de comportamiento alimenticio (Pérez \& Bencomo, 2015).

Estos hábitos alimentarios han sido más afectados durante la pandemia por COVID-19, al parecer los consumidores han tendido a preferir dietas menos nutritivas, menos frescas y más económicas. Dicha decisión podría explicarse por una disminución significativa del ingreso económico y las restricciones a la movilidad impuestas para evitar la propagación del coronavirus (CEPAL, 2020).

Una mala nutrición supone mayores riesgos de contraer enfermedades. En América Latina y el Caribe hemos convivido en tiempo recientes con alarmantes cifras de malnutrición por sobrepeso, obesidad y desnutrición. Un problema de salud pública aún más graves en tiempo de pandemia (CEPAL, 2020). Según cifras de la Organización Mundial de la Salud, ya la población mundial cerca de 35 millones de personas moría cada año por enfermedades crónicas no transmisibles, que son ocasionadas por una alimentación inadecuada, además se afirma que un aproximado de $5 \%$ de estas muertes corresponden a personas en edad estudiantil a consecuencia de los hábitos alimenticios que tiene la población en relación con el estado nutricional, lo que conlleva la presencia de enfermedades metabólicas (Cedeño \& Cevallos, 2017).

Por ende, estos malos hábitos alimenticios pueden ser un importante factor de riesgo para el desarrollo de enfermedades como obesidad, enfermedad coronaria, cáncer, diabetes, entre otras (FAO, 2003). Actualmente la prevalencia de estas enfermedades ha aumentado de manera alarmante a nivel mundial, de manera que anualmente las enfermedades crónicas no transmisibles causan 35 millones de muertes, $75 \%$ de las cuales se producen en países de ingresos económicos bajos y medios (OMS, 2016).

La Organización Mundial de la Salud ha estimado que el $16.23 \%$ de las mujeres y el $3.3 \%$ de los 
hombres entre 12 y 24 años, sufren algún tipo de trastorno alimentario. Sin embargo, a nivel mundial se observa una alta prevalencia de hábitos alimentarios inadecuados; siendo la prevalencia de consumo de comidas rápidas en jóvenes para el año 2011 se evidencio que el 17\% se da en Europa, $36 \%$ en Asia Pacifico y $47 \%$ con mayor relevancia se observa en América Latina (Moneo, Sirgado, \& Lamas, 2016).

A partir de ese año se comprobó que la inseguridad alimentaria moderada o grave en América Latina se incrementó de forma considerable. La inseguridad alimentaria medida a partir de la escala de experiencia de inseguridad alimentaria, paso de $26.2 \%$ a $31.3 \%$ entre los trienios 2014 - 2016 y 2016 -2013. Este aumento produjo que más de 32 millones de personas se sumaran a los casi 155 millones que vivían son inseguridad alimentaria en el 2014 - 2016 (FAO, 2019).

Ecuador no se escapa de dicha situación, debido a que los hábitos alimenticios es uno de los principales problemas que enfrenta el ministerio de salud pública por lo consiguiente: Manabí es una de la provincia que por años se observa un desbalance nutricional de los adolescentes y actualmente estos han sido mayormente afectados por la crisis sanitaria que se vive hoy en día (Lucas, 2018).

Según la Encuesta Nacional de Salud y Nutrición del Ministerio de Salud y el Instituto Nacional de Estadística y Censo (INEC), en Ecuador el 29.2\% de la población de 5 a 29 años consume excesivamente carbohidratos y un $6 \%$ grasas. La prevalencia destaca que el $60 \%$ de hombres y el $65.5 \%$ en mujeres, que empiezan a consumir de forma inadecuada sus alimentos es a partir de los 15 años. Por consiguiente, en Manabí según reportes de la dirección de salud señala: que las personas que presentan a consecuencia de sus hábitos alimenticios problemas cardiovasculares un $18 \%$ relacionados con la hipertensión arterial y el $21 \%$ con diabetes (Cedeño \& Cevallos, 2017).

El COVID-19 ha desencadenado muchas modificaciones en el día a día, el distanciamiento y confinamiento social es una de las más notorias, como consecuencia de esto muchas familias que frecuentemente salían a comer, que no cocinan o tenían personal que les elaboren sus alimentos son los más afectados en la crisis (ALvarado \& Barros, 2016). Estudios revelan que evaluar la seguridad alimentaria es de gran importancia por las repercusiones en el estado de la salud, la nutrición de los individuos y la capacidad con la que en cada hogar acceden a los alimentos para llevar una vida saludable, sin estar en riesgo de inseguridad y de vulnerabilidad de cada miembro del hogar a la misma (Pico \& Pachón, 2012).

En todo el mundo las dietas insalubres y la falta de actividad física están entre los principales factores de riesgo para la salud. Una dieta saludable y
Vol. 4, Nro. 1, Publicado 2021-06-30

equilibrada nos permitirá mantenernos $\mathrm{y}$ protegernos de enfermedades no transmisibles, donde muchas de estas están asociadas al COVID19 con un empeoramiento en los síntomas (ALvarado \& Barros, 2016). Se busca conocer las modificaciones y las practicas alimentarias como resultado del confinamiento, esto nos permitirá formar un diagnóstico de los posibles riesgos que puede tener dicha población. Así mismo las conductas de alimentación, estilo de vida saludable y seguridad alimentaria que adopten nos permitirá analizar los efectos negativos que ha desencadenado el aislamiento (ALvarado \& Barros, 2016).

Es muy importante que la población en general cuente con directrices o pautas que les ayuden en su orientación para obtener una alimentación adecuada y lleven un estilo de vida saludable. Fomentar el mantenimiento de una salud optima por medio de una alimentación completa para evitar un empeoramiento de enfermedades crónicas existente o no existente (ALvarado \& Barros, 2016).

El objeto de estudio de la investigación fue los hábitos alimenticos. El campo la salud publica en la rama de la nutrición, se estableció como objetivo general: Identificar los hábitos alimenticios en los adolescentes de 13 a 19 años de edad del centro de salud Junín en tiempos de pandemia. Como objetivos específicos: Investigar los datos sociodemográficos de los adolescentes de 13 a 19 años en el centro de salud Junín; Describir los hábitos alimenticios aplicando el cuestionario de frecuencia de consumo de alimentos (CCFA) en los adolescentes de 13 a 19 años en el centro de salud Junín; y, Determinar si la pandemia afecta de manera directa los hábitos alimenticios en los adolescentes de 13 a 19 años en el Centro de Salud. Materiales y métodos

Para la realización de este proyecto se consideró a la población adulta joven, es decir, adolescentes de 13 a 19 años que han sido atendidos en el centro de salud Junín. Se realizó un estudio descriptivo de corte transversal. Se aplicó el cuestionario ya validado de frecuencia de consumo de alimentos que fue diseñado para ser aplicado en adolescentes y adultos; de igual manera se anexo preguntas al cuestionario para identificar si estos han sido afectados por la pandemia a nivel mundial, concluyendo la investigación con una entrevista dirigida a un profesional de salud especializado en el campo de la nutrición.

\section{Diagnóstico o estudio de campo}

La investigación se realizó con una muestra de 200 adolescentes, que correspondían a la población ya descrita, escogiendo como muestra a toda la población para este estudio, se aplicó una encuesta validada y revisada por el docente tutor de la investigación, el cuestionario está compuesto por 18 preguntas orientadas a conocer detalles 
específicos de cómo es su frecuencia de consumo de alimentos y si estos hábitos alimentarios han sido afectados durante la pandemia, así mismo se aplicó una entrevista dirigida a un profesional de la
Vol. 4, Nro. 1, Publicado 2021-06-30

salud experta en la rama de la nutrición. Según las encuestas aplicada a la población seleccionada se obtuvieron los siguientes resultados:

Tabla 1. Datos sociodemográficos de los adolescentes que pertenecen al centro de salud Junín

\begin{tabular}{|c|c|c|c|c|c|c|}
\hline \multirow{2}{*}{ Orden } & & \multirow{2}{*}{ Alternativas } & \multirow{2}{*}{$\mathbf{F}$} & \multirow{2}{*}{$\%$} & \multicolumn{2}{|c|}{ Total } \\
\hline & & & & & $\mathbf{F}$ & $\%$ \\
\hline \multirow{2}{*}{\multicolumn{2}{|c|}{ Género }} & Masculino & 96 & 48,00 & \multirow{2}{*}{200} & \multirow{2}{*}{100,00} \\
\hline & & Femenino & 104 & 52,00 & & \\
\hline \multirow{3}{*}{\multicolumn{2}{|c|}{ Edad }} & 13 a 15 años & 108 & 54,00 & \multirow{3}{*}{200} & \multirow{3}{*}{100,00} \\
\hline & & 16 a 18 años & 86 & 43,00 & & \\
\hline & & 19 años & 6 & 3,00 & & \\
\hline \multirow{5}{*}{\multicolumn{2}{|c|}{ Estado Civil }} & Soltero & 200 & 100,00 & \multirow{5}{*}{200} & \multirow{5}{*}{100,00} \\
\hline & & Casado & 0 & 0,00 & & \\
\hline & & Unión de hecho & 0 & 0,00 & & \\
\hline & & Viudo & 0 & 0,00 & & \\
\hline & & Divorciado & 0 & 0,00 & & \\
\hline \multirow{2}{*}{\multicolumn{2}{|c|}{$\begin{array}{l}\text { Lugar } \\
\text { procedencia }\end{array}$}} & Urbana & 166 & 83,00 & \multirow{2}{*}{200} & \multirow{2}{*}{100,00} \\
\hline & & Rural & 34 & 17,00 & & \\
\hline
\end{tabular}

Por medio de la encuesta aplicada, en la tabla 1 se aprecia el predominio del género femenino $(52.00 \%)$ esto se debe a que por estar en su pleno desarrollo son las que más ameritan revisión médica, así mismo respecto a la edad se observa que el $54.00 \%$ se encuentra en un rango de 13 a 15 años pudiendo deducir que a esa edad las madres suelen llevarle control más seguido a sus representados, en cuanto al estado civil el $100 \%$ fueron solteros, debido a que son personas jóvenes y que se encuentran estudiando, así mismo el lugar de procedencia que predomina es el sector urbano $(83.00 \%)$ este puede ser uno de los motivos que afecte la alimentación de los adolescente entre 13 a 19 años, ya que por motivos de la pandemia el sector urbano se vio más afectado que la zona rural.

Tabla 2. Alimentación de los adolescentes durante la pandemia

\begin{tabular}{lll} 
Alternativa & $\mathbf{F}$ & $\boldsymbol{\%}$ \\
\hline Excelente & 0,00 & 0,00 \\
\hline Muy Buena & 17,00 & 8,50 \\
\hline Buena & 32,00 & 16,00 \\
\hline Regular & 92,00 & 46,00 \\
\hline Deficiente & 59,00 & 29,50 \\
\hline Total & $\mathbf{2 0 0 , 0 0}$ & $\mathbf{1 0 0 , 0 0}$ \\
\hline
\end{tabular}

De acuerdo a los resultados de la encuesta, se observa que la alimentación de los adolescentes durante la pandemia fue regular, muy probablemente se deba a que por el confinamiento los padres no podían salir a trabajar, ya que la mayoría de ellos se dedican al comercio ambulante y por ende el ingreso económico iba a ser muy 
bajo, siendo uno de los motivos por el cual la alimentación se debió ajustar a las condiciones en que cada familia se vio inmersa.

Tabla 3. Alimentación equilibrada de los adolescentes

\begin{tabular}{lll}
\hline Alternativa & F & \% \\
\hline Excelente & 0,00 & 0,00 \\
\hline Muy Buena & 5,00 & 2,50 \\
\hline Buena & 58,00 & 29,00 \\
\hline Regular & 101,00 & 50,50 \\
\hline Deficiente & 36,00 & 18,00 \\
\hline Total & $\mathbf{2 0 0 , 0 0}$ & $\mathbf{1 0 0 , 0 0}$ \\
\hline
\end{tabular}

Los adolescentes encuestados manifestaron que han tenido dificultad en el consumo de alimentos equilibrados, probablemente por la crisis que atraviesa el país hace que los alimentos sean racionados y muchas veces sean suprimidas comidas rápidas, comidas no preparadas en casa o simplemente comida denominada "chatarra", identificando este indicador como uno de los causantes del problema de estudio en los adolescentes quienes deben tener una mejor alimentación para su normal desarrollo.

Tabla 4. Consumo diario de frutas, legumbres y verduras de los adolescentes

\begin{tabular}{lll}
\hline Alternativa & F & \% \\
\hline Excelente & 0,00 & 0,00 \\
\hline Muy Buena & 11,00 & 5,50 \\
\hline Buena & 49,00 & 24,50 \\
\hline Regular & 132,00 & 66,00 \\
\hline Deficiente & 8,00 & 4,00 \\
\hline Total & $\mathbf{2 0 0 , 0 0}$ & $\mathbf{1 0 0 , 0 0}$ \\
\hline
\end{tabular}

En la tabla 4 se observa que el consumo diario de frutas, legumbres y verduras es muy irregular en los adolescentes de 13 a 19 años de edad atendidos en el centro de salud Junín, este desequilibrio en la alimentación pone en riesgo el estado de salud de los adolescentes, pudiendo provocar obesidad o enfermedades cardiovasculares, en este sentido, en la adolescencia es el momento ideal para la promoción de la salud del adulto, con la instauración de hábitos de vida y una alimentación balanceada (Marugán, Sanz, Monasterio, \& Pavón, 2018).

Tabla 5. Consumo de alimentos ocasionales o chatarra de los adolescentes en la pandemia

\begin{tabular}{lll} 
Alternativa & F & \% \\
\hline Excelente & 0,00 & 0,00 \\
\hline Muy Buena & 0,00 & 0,00 \\
\hline Buena & 0,00 & 0,00 \\
\hline Regular & 36,00 & 36,00 \\
\hline
\end{tabular}


De los resultados obtenidos, en la tabla se puede observar que una gran parte de los encuestados durante la pandemia fue consumieron alimentos ocasionales o comida chatarra, muy probablemente sea por la prohibición que dio el gobierno de salir de las casas algo beneficioso para el adolescente ya que el organismo de ellos necesita en su gran mayoría vitaminas y minerales, que aporten energía y vitalidad para su desarrollo normal, pero al consumir estas comidas ocasionales o chatarra, el organismo corre el riesgo de enfermarse, por sus grandes cantidades de sodio, el cual puede elevar de manera considerables la presión arterial ocasionando problemas en el corazón o diabetes e incluso pudiendo contraer cáncer (Mora, 2019).

Tabla 6. Frecuencia de la alimentación diaria de los adolescentes en la pandemia

\begin{tabular}{lll} 
Alternativa & F & \% \\
\hline $1-2$ veces & 76,00 & 38,00 \\
\hline $3-4$ veces & 124,00 & 62,00 \\
\hline 5 o más veces & 0,00 & 0,00 \\
\hline Total & $\mathbf{2 0 0 , 0 0}$ & $\mathbf{1 0 0 , 0 0}$ \\
\hline
\end{tabular}

De los resultados obtenidos, se puede observar que la frecuencia de la alimentación diaria de los adolescentes estuvo entre los rangos normales de la comida, aun sabiendo que dentro de estos platos de comida los alimentos no eran los adecuados para el crecimiento de los adolescentes.

Tabla 7. Actividad física de los adolescentes de la pandemia

\begin{tabular}{lll} 
Alternativa & F & \% \\
\hline No realizaba actividad física & 109,00 & 54,50 \\
\hline 2 - 3 veces por semana & 38,00 & 19,00 \\
\hline 4 - 5 por semana & 12,00 & 6,00 \\
\hline Todos los días & 41,00 & 20,50 \\
\hline Total & $\mathbf{2 0 0 , 0 0}$ & $\mathbf{1 0 0 , 0 0}$
\end{tabular}

La gran mayoría de los adolescentes durante la pandemia no realizaron actividad física, siendo uno de los indicadores muy desfavorable para los adolescentes, ya que la actividad física puede ayudar a los adolescentes a sentirse más lleno de energía, a mejorar la concentración y atención, a mantener un peso saludable, así como prevenir enfermedades cardiacas, la diabetes y otros problemas de salud en el futuro (Gavin, 2019).

\section{Conclusiones}

Las características sociodemográficas que enmarcan a la población de los adolescentes de 13 a 19 años, la mayoría son de género femenino en relación al masculino, concentrados en un mayor porcentaje en el rango de edad entre 13 a 15 años, el estado civil predominante es soltero/a, entre estos datos cabe resaltar la importancia del monitoreo de estos pacientes en la atención primaria.

El mayor problema de los hábitos alimenticios de los adolescentes es el poco conocimiento al momento de la elección de los alimentos, y la poca frecuencia de consumo de alimentos nutritivos y variados que no aportan nutrientes y proteínas para el normal desarrollo de los adolescentes.

Los factores de riesgos que influyen en los hábitos alimenticios de los adolescentes son los factores socioeconómicos, debido a la pandemia la 
situación económica ha sido la que más afectada ha estado dentro de los senos familiares, provocando
Periodo. Enero - Junio 2021

Vol. 4, Nro. 1, Publicado 2021-06-30

que la alimentación no compense lo que organismo necesita para un correcto funcionamiento.

\section{Bibliografía}

ALvarado, T. M., \& Barros, B. A. (2016). Hábitos alimentarios, estado nutricional de los estudiantes de la Unidad Educativa Manuel Córdova Galarza. Tesis de licenciatura, Universidad de Cuenca , Nutrición y Dietética, Cuenca.

Cedeño, P. L., \& Cevallos, Z. M. (2017). Estado nutricional y hábitos alimentarios de los estudiantes del I Y II nivel de la escuela de medicina. Tesis de titulación, 2017, Departamento de nutrición y dietética, Portoviejo.

CEPAL. (2020). Nutrición. Recuperado el 2021 de marzo de 2021, de CEPAL: https://www.cepal.org/es/publicaciones/45794-sistemas-alimentarios-covid-19-americalatina-caribe-ndeg-10-habitos-consumo

FAO. (2003). Expert consultation on diet, nutrition, and the prevention of chronic diseases, FAO. Recuperado el 27 de marzo de 2021, de World haelth organization: https://scielo.conicyt.cl/scielo.php?script=S0717-75182016000200006

FAO, O. W. (julio de 2019). Hacia entornos alimentarios mas saludables que hagan frente a todas las formas de malnutricion. Panorama de la seguridad alimentaria y nutricional en américa latina y el caribe., I(2).

Gavin, M. I. (2019). Recuperado el 26 de Marzo de 2021, de KidsHealth: https://kidshealth.org/es/parents/fitness-13-18-esp.html

Lucas, J. J. (2018). Hábitos alimentarios, actividad física y salud en los estudiantes de la carrera de pedagogía de la imagen y la literatura de la facultad de ciencia de la educacion de la ULEAM. Tesis de titulación, Universidad Laica Eloy Alfaro de Manabí, Carrera de educación física, deportes y recreación, Manta.

Martínez, C. V., Cobo, J., \& Carbajal, A. (2011). Recuperado el 27 de maro de 2021, de Evaluación del estado nutricional de un grupo de estudiantes universitarios mediante parámetros dietéticos y de composición corporal: http://scielo.iscii.es/scielo.php?script=sci:arttext\&pid=S0212$16112005000400006 \&$ Ing=es

Marugán, J. M., Sanz, M., Monasterio, C. L., \& Pavón, M. d. (Junio de 2018). Alimentación en el adolescente. Protocolos diagn "ostico de gastroenterología, hapatología y nutricion pediátrica, $/ /(312)$.

Ministerio de Salud Pública del Ecuador. (2012). Recuperado el 26 de marzo de 2021, de Guias Alimentarias para los Adolescentes de la Sierra. Sociedad Ecuatoriana de Ciencias de la Alimentación y Nutrición: http://liceodeloja.org/contenidos/index.php?view=article\&catid=5\%3Anoticias \&id=172\% Aguias-alimentariaspara'los'adolescentes'de'la'sierra\&format=pdf\&option=com_content\&ltemid=5

Moneo, J., Sirgado, M., \& Lamas, A. (2016). Recuperado el 27 de marzo de 2021, de Fast food consumption: http://www.abc.es/gestor-documental/uploads/Sociedad/comidarapida.pdf 
Mora, T. (2019). Recuperado el 26 de Marzo de 2021, de Consumo de comida chatarra en adolescentes: https://www.vix.com/es/imj/familia/5615/consumo-de-comida-chatarraen-adolescentes

Morales, L. H. (2020). Emergencia sanitaria por COVID-19 y su impacto en los hábitos alimenticios. Trabajo de titulación, Universidad Católica de Santiago de Guayaquil, Facultad de Medicina, Guayaquil, Ecuador.

OMS. (2016). Recuperado el 27 de marzo de 2021, de Non communicable diseases: http://www.who.int/mediacentre/fastsheets/fs355/es/

Organización Mundial de la Salud. (2016). Recuperado el 27 de marzo de 2021, de Temas de nutrición: Nutrición.

Pacheco, R. M., Michelena, G. M., Mora, G. R., \& Miranda, G. O. (abril - junio de 2014). Calidad de vida relacionada con la salud en estudiantes universitarios. Revista cubana de medicina militar, 43(2).

Pérez, A., \& Bencomo, M. (2015). Hábitos alimeticios de los estudiantes universitarios. Tesis doctoral, Universidad de Caraboro, Departamento de orientación, Barbula.

Pico, F. S., \& Pachón, H. (2012). Factores asociados con la seguridad alimentaria en un Municipio Rural del norte del Cauca,. Archivos Latinoamericanos de Nutrición, 62(3)(227-233).

PUCE, Escuela de medicina. (2015). Recuperado el 21 de marzo de 2021, de Evaluacion del estado nutricional. 